\title{
Analysis of the Vlan Network Delay Performance to Improve Quality of Services (QOS)
}

\author{
Walaa Amayreh \\ Yarmouk University \\ Jordan, Irbid
}

\author{
Norah Alqahtani \\ Salman Bin Abdulaziz \\ University \\ $\mathrm{KSA}$, al-kharj
}

\author{
Badryah Al-Balawi \\ Tabuk University \\ KSA, Tabuk
}

\begin{abstract}
Technological revolution that has conquered the world and the In the modern life, the internet network become important part in the life because it provides many effective services in many fields that leading the responsible Categories to interested in improve quality of services (QOS) for the network.

There are increasing in using internet networks and many new modern services access in the life fields that leading to emergence of many problems and challenges in network. The delay is the most effected in the network because it caused reducing in QOS.
\end{abstract}

The real time application is modern services such as voice and video which needing more time and effort to transfer data in the network because it have large data that making it to be the most effect applications by delay.

A large number from network studding interested in create new technical to improve QOS in local area networks and wide area networks that using different technical methods. This study interested in showing the importance in using VLAN technology to improve QOS and it refers to its role in reducing the delay in the network.

The structures of network when building it or improving it that is more important and sensitive thing that effected in ability it in providing the services in effective way. That referred to manage the networks and divided it into parts helping to transfer data in direct way to selected partition not all. Vlan technology mean divided the local area network in to many departments to give the security for each departments and acceleration of the arrival of the data, which sent to it. That in clearly referring to overcome the problem of delay when using VLAN technology and improve QOS for the networks.

\section{Keywords}

Delay, QOS, Local Network, VLAN, Performance, VPN, AODV and network design.

\section{INTRODUCTION}

Quality of service is a measure of network performance for all services which providing from the network. The Improving performance for the networks is the most important goals because it has a direct impact on all the services, which providing by the network therefore, when building any network that important to building it in effective way to improve QOS for it by reducing delay Rate value.
There are many technical methods and technologies to increase the ability it and improve its performance arriving high quality of services (QOS) level. The reaching the desired level of network performance means solving many of the problems and challenges that facing the networks and developing activities important services in the many of fields in the life.

The use of the internet in an effective way and manages the networking to Invests every part of networks helping to improve QOS for the networks in easy and not cost way.

The delay is formed a large scale from network problems which particular appear in real time application from video and voices. There are a large trend towards Real time services in these days, which led to the emergence of a real problem in this area.

The study interested in improve QOS for network in effective and easy way using VLAN technology which cover local area network by overcome delay problem and The organization of the network and improve the built.

The idea of the project can be applied by building more than one network, studying and analysis of the performance of these networks in data transmission, including the study of the quality of their performance, in some services which selecting it, and note how the delay effected in its performance.

The project start by researching to apply the idea using OPNET program, and improve the QOS when using Vlan technology in the network, showing how the delay effect on the quality of services which providing by the vlan network.

The project interested in analyses the performance many of services, which Affected by delays especially voices, and videos. Building and analyzing the project idea will be applied using OPNET simulator to show how selecting good way in designing network to reduce the value delay for improving the QOS.

\section{RESEARCH PROBLEM}

The poor performance of networks and the lack of quality of services put the internet network in real problem and the delay in real time application is formed the main reason in reduce the QOS especially when the users who using this application increasing in constantly.

The research interested in determine how improving QOS and designing network in effective and easy way using VLAN technology. Mange networks to overcome delay problem is formed the main step to apply the main aims in improving QOS. The building of networks in active method to support 
the continued increasing in the number of Internet users making the networks provide services in high level for long time.

\section{RELATED PREVIOUS STUDIES}

The information's shown below are the Research into sources of information, Found during the research stage of contain the related information to the main project. These resources were carefully chosen to be used in answering the academic question as they hold relevant and necessary information and studies about the effect of delay in vlan network using OPNET simulator analysis tool. These resources were all found to have the kind of information needed to address and to develop the final artefact of the project and the final report.

The delay plays an active role in the network as negative way because that reduce the quality of services for network so there are many studies that interested in delay sensitive and how improve quality of services in the network.

The delay is the main important characteristics of networks; the delay mean the lost time, which used in network to transfer data from node to other, despite of that the time it takes simple fractions of a second in the habit, but it caused some problems in many networks in multiple cases. [1]

Delays is effect in network and lead to reduce its quality of services for it in their performance in clearly. However, the delay value is different from network to other that related to many of criteria such as type of services, the real time application is one of the services most affected from delay. [2]
Real time application is formed large part from the modern services in the life and it be effected by delay in clearly such as voip (voice over Internet Protocol). The result performance in the network, which provide voip and video services show the need to improve the performance in it. [3]

The quality of services (QOS) is expresses about the performance for network and its ability to provide the services. QOS is more important object in transfer data from network to other and evaluation of existing networks in many services, which provided in it. [4]

Vlan Abbreviation for the concept virtual local area network that express about local network contain from server, workstation and other network devices that appear in the same LAN. [5] Vlan technology used to divide the same network in to many virtual LAN network in departments to provide the security for the network. [6]

Several studies indicate that Vlan is modern technical used in local network to manage it and provide the security for network contents but there is no a clear signal about the use Vlan technology in improve QOS for network by Reduce the value of the delay.

\subsection{Comparative analysis}

The table show some technical that interested in improve the QOS in the network in many methods and technical. It showing the importance in using VLAN because it easy way and not cost in local network.

Table 1. Comparative analysis of existing techniques

\begin{tabular}{|c|c|c|c|}
\hline Title Study & Technical Method & Idea process shape & In comparison \\
\hline " MPLS network management ".[7] & $\begin{array}{l}\text { Multiprotocol Label Switching (MPLS) } \\
\text { is a technology that provide a } \\
\text { mechanism to improve QOS. It uses the } \\
\text { information embedded in the labels } \\
\text { attached to the IP packets. }\end{array}$ & Label swapping & $\begin{array}{l}\text { It provide good performance } \\
\text { in real time application but it } \\
\text { is high cost }\end{array}$ \\
\hline $\begin{array}{l}\text { " AOD } 2 \text { V: Ad Hoc On Demand and } \\
\text { Delay Constrained Distance Vector } \\
\text { Routing for mobile sensor/actor } \\
\text { networks ".[8] }\end{array}$ & $\begin{array}{l}\text { Ad-hoc On-demand Distance Vector } \\
\text { (AODV) interested in find the shortest } \\
\text { path in the network without Attention to } \\
\text { QOS or accuracy for the network. }\end{array}$ & & $\begin{array}{l}\text { it improve QOS in many } \\
\text { services but it have problem in } \\
\text { real Time application }\end{array}$ \\
\hline $\begin{array}{l}\text { "VPN: Virtual private network, } \\
\text { tunnel, ipsec, layer } 2 \text { tunneling } \\
\text { protocol, mobile".[9] }\end{array}$ & $\begin{array}{l}\text { virtual private network provide security } \\
\text { (VPN )for network in internet } \\
\text { communication }\end{array}$ & VPN connection & $\begin{array}{l}\text { Improve QOS but it is it } \\
\text { complex way }\end{array}$ \\
\hline $\begin{array}{l}\text { "Analysis Of The Vlan Network } \\
\text { Delay Performance To Improve } \\
\text { Quality Of Services (QOS)". } \\
\text { [project study] }\end{array}$ & $\begin{array}{l}\text { Virtual local area network is simple and } \\
\text { effective technical that providing the } \\
\text { security for network and mange that } \\
\text { into many departments to improve it by } \\
\text { reduce the delay in it. }\end{array}$ & Dep1 & $\begin{array}{l}\text { Perfect way to overcome } \\
\text { delay in many cases for the } \\
\text { network and improve QOS } \\
\text { with Low Cost. }\end{array}$ \\
\hline
\end{tabular}




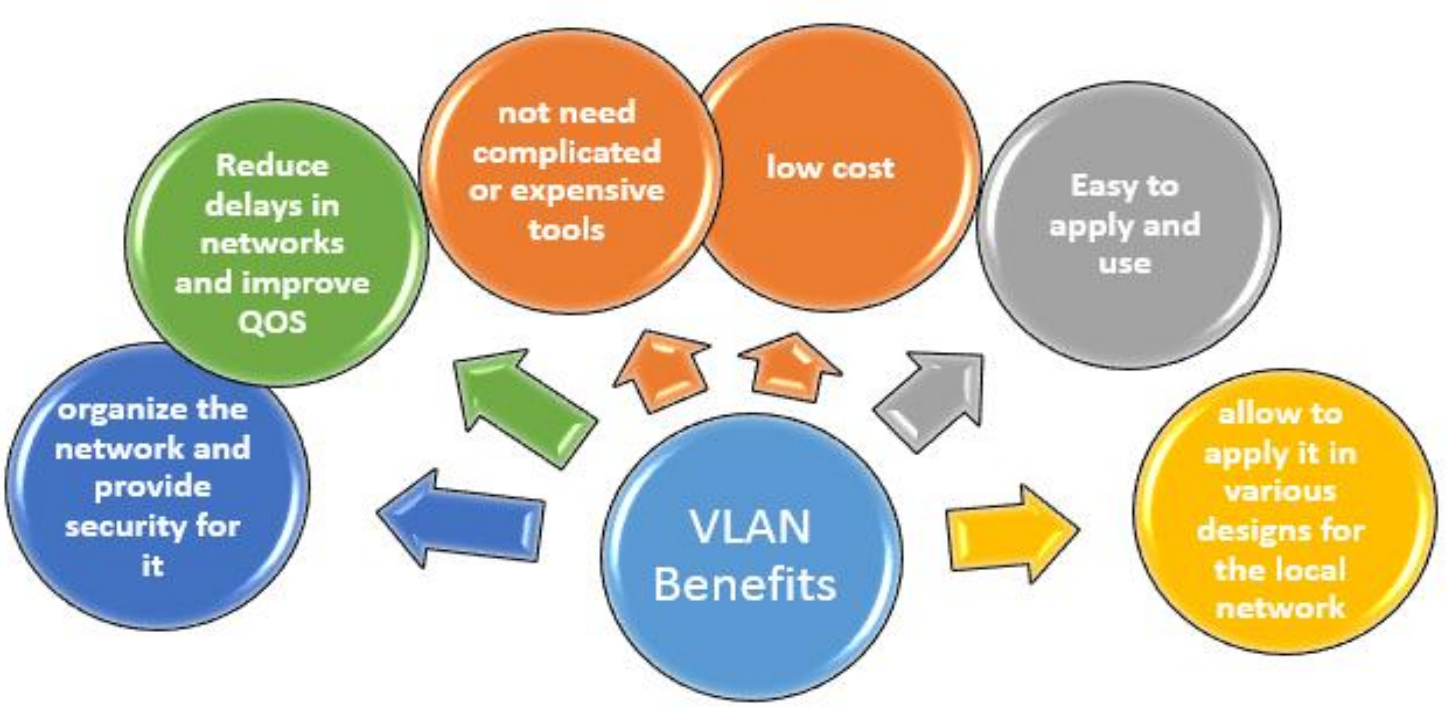

Fig 1: benefits VLAN which make the network more effective and efficient

\section{RESEARCH HYPOTHESIS}

- How to reduce the delay to get high performance in the network?

- How can improve the QOS when using Vlan technology in the network?

\section{AIMS}

The aim of the project is to study the effect of virtual LAN (VLAN) networks in improving the performance for network. In addition, to know an effective ways increasing the quality of services provided by the network and how reducing effected delay in the network.

\section{OBJECTIVES}

In following points showing the main objectives from this research:

- Building the networks in active method to support the continued increasing in the number of Internet users.

- Study the benefit of using VLAN technology in designing a network.

- $\quad$ Analyze the delay-sensitive services performance.

- know what type of traffic have the most impact on QOS

\section{PROCEDURES AND METHODOLOGIES}

The project idea is applied by designing local network using Vlan technology. the network cab be built using many simulators such as OPNET simulator, Riverbed modeler program and so on but the OPNET simulator is the best program to apply many ideas in network word to analysis the performance for any network.

All tools which needing it for the design are available in OPNET simulator that allow to design and configuration in it. All Characteristics were selected for all the services by program using "choose individual DES statistics". In addition, show the performance for all that in various forms of curves provided by the Riverbed program.

The main idea in the project related to build network with apply vlan technology. The vlan (Virtual local area network) technology is used to improve quality of services for the network, it organize data transmission in the network.

It means division the same local network in to multiple small networking called departments to give each part security and to protect them to store all its data.

To study the performance the network will be used VLAN system to test many parameters showing the result to compare performance between any selected scenarios. In a simple way, to apply the idea the network will be designed in two scenarios; the first scenario interested in using vlan technology and the second scenario not using vlan technology.

Each scenario consists of two networks to study the performance for transfer data between these networks. The performance will cover some applications to study the behavior and analysis it. That following many QOS parameters, studying the performance include many Characteristics such as Packet Delay variation, Packet EndTo-End Delay (sec),Traffic Received(byte/sec),Traffic Received(packet/sec),Traffic Sent(byte/sec) and Traffic Sent (packet/sec) but the delay informed the main Characteristic in the study.

The study interested in application that effected by delay in clearly. The real time application is more effected in delay so there are more important to study real application such as voice (VoIP application) and video (video conferencing) application.

\section{CONCLUSION}

The delay is the main reason, which caused many problems and challenge in the network that reducing the QOS for network. There are many technical methods used to overcome in it. Network building and organizing an effective way to solve the delay problem. Organization and division translate the traffic is one of the most important ways to improve the performance of networks. Vlan technology is apply that idea 
because it interested in division LAN network for departments. That organize the traffic from and to network so the VLAN technology is effective, not expensive and perfect way to overcome delay in many cases for the network.

\section{EXPERIMENTAL ANALYSIS}

The idea of the project had been applied by design simple network in two scenarios contain from two departments: engineering and financial. The network that using VLAN technology give results better than the network without using VLAN technology, for example the delay value in first scenario without using VLAN almost equal from .0031 to .0036 second But in second scenario with using VLAN almost equal from .0026 to .0032 second, the details in fig 2 . That proving the role of using VLAN technology in Reducing delays and improving the performance for the network to improve QOS.

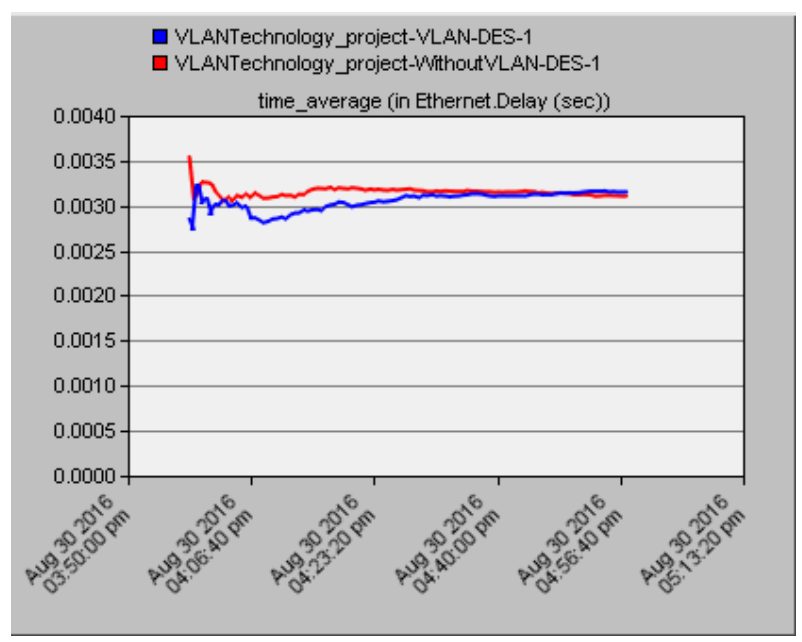

Fig 2: Time Average (in Ethernet Delay (Sec))

\section{ACKNOWLEDGMENTS}

All respect and appreciation to the friends and the parents who are the first motivation for success and progress. More love and thanks for team work who help to arrive the success of this paper and published.

\section{REFERENCES}

[1] Schöbel, A. (2006). Optimization in public transportation: Stop location, delay management and tariff zone design in a public transportation network. New York: Springer.

[2] Jeffay, K., \& Zhang, H. (2002). Readings in multimedia computing and networking. San Francisco: Morgan Kaufmann.

[3] Pitts, J. M., Schormans, J. A., \& Pitts, J. M. (2000). Introduction to IP and ATM design and performance: With applications analysis software. Chichester: J. Wiley.

[4] Hardy, W. C. (2001). QoS: Measurement and evaluation of telecommunications quality of service. Chichester: Wiley.

[5] Bernardini, A. (2004). Vlan. Quimper: Le Quartier.

[6] Blacharski, D. (1998). Network security in a mixed environment. Foster City, CA: IDG Books Worldwide.
[7] Nadeau, T. (2003). MPLS network management: MIBs, tools, and techniques. San Francisco, Calif.: Morgan Kaufmann.

[8] Sama, A. R. (2008). AOD ${ }^{2}$ V: Ad Hoc On Demand and Delay Constrained Distance Vector Routing for mobile sensor/actor networks.

[9] Wikipedia, Q. (2013). Vpn: Virtual private network, tunnel, ipsec, layer 2 tunneling protocol, mobile. Place of publication not identified: University-Press Org.

[10] Blacharski, D. (1998). Network security in a mixed environment. Foster City, CA: IDG Books Worldwide.

[11] Elahi, A., \& Elahi, M. (2006). Data, network, and Internet communications technology. Clifton Park, NY: Thomson Delmar Learning.

[12] Rooney, S., Hörtnagl, C., \& Krause, J. (1999). Automatic VLAN creation based on on-line measurement. Yorktown Heights, NY: IBM T.J. Watson Research Center.

[13] Chowdhury, D. D. (2000). High speed LAN technology handbook. Berlin: Springer .

[14] Donoso, Y. (2009). Network design for IP convergence. Boca Raton: CRC Press.

[15] Trivedi, B. (2013). Computer networks. New Delhi, India: Oxford University Press.

[16] Saad, T. W. (2002). Support of delay sensitive applications over MPLS and differentiated services enabled networks.

[17] Jiménez, D. A. (2002). Delay-sensitive branch predictors for future technologies.

[18] Fu, F. (2010). A unified framework for delay-sensitive communications.

[19] Gaïti, D. (2006). Network control and engineering for QoS, security and mobility, V: IFIP 19th World Computer Congress, TC-6, 5th IFIP International Conference on Network Control and Engineering for QoS, Security and Mobility, August 20-25, 2006, Santiago, Chile. New York: Springer.

[20] Saad, T. W. (2002). Support of delay sensitive applications over MPLS and differentiated services enabled networks.

[21] Hardy, W. C. (2001). QoS: Measurement and evaluation of telecommunications quality of service. Chichester: Wiley.

[22] Rocha, A. (2013). Advances in Information Systems and Technologies. Heidelberg: Springer.

[23] ] Xiao, X. (2008). Technical, commercial, and regulatory challenges of QoS. Amsterdam: Elsevier / Morgan Kaufmann.

[24] Michelli, D., \& McWilliams, F. (1996). Networking. Corby: Institute of Management.

[25] Lurch, T., Messervy, P., \& Tangitu, P. (1989). Networking. Wellington, N.Z.: Health Services Equal Employment Opportunities Development Unit. 